\title{
Optic Nerve Head and Retinal Nerve Fiber Layer Imaging in Glaucoma Diagnosis
}

\author{
${ }^{1}$ Rajul Parikh, ${ }^{2}$ Shefali Parikh, ${ }^{1}$ Kulin Kothari \\ ${ }^{1}$ Bombay City Eye Institute and Research Center, Mumbai, Maharashtra, India \\ ${ }^{2}$ Lotus Eye Hospital, Mumbai, Maharashtra, India
}

Glaucoma is a chronic, progressive neurodegenerative disease. It is defined as chronic progressive optic neuropathy with typical optic disk and retinal nerve fiber layer (RNFL) changes with corresponding visual field defect. Intraocular pressure (IOP) is considered only a major risk factor and not a diagnostic criterion.

At present, clinically visible RNFL defects are considered as the sensitive indicator for early diagnosis of glaucoma. Experimental studies have shown, that localized RNFL defects can ophthalmoscopically be detected if more than $50 \%$ of the thickness of the retinal nerve fiber layer is lost. ${ }^{1,2}$ The gold standard for detection of the functional damage in glaucoma is automated perimetry. It has been reported that locations with a $5 \mathrm{~dB}$ decrease in sensitivity on WWP (white-on-white perimetry) already had a $20 \%$ loss of retinal ganglion cells; this was $40 \%$ at locations with a $10 \mathrm{~dB}$ decrease in sensitivity. As the optic nerve and RNFL damage is irreversible, the early diagnosis of glaucoma is important.

Retinal ganglion cell loss is detected as:

a. Thinning of the RNFL (RNFL defect)

b. Changes in the shape of the $\mathrm{ONH}$ (neuroretinal rim loss).

Currently, optic disk photography is considered gold standard to detect changes to the optic nerve head. Photographs should be taken at baseline and at follow-ups. However, for routine clinical use optic disk photography has several limitations. With the advent of high-resolution new imaging technologies like HRT (confocal laser ophthalmoscope), GDx VCC (scanning laser polarimetry), OCT (optical coherence tomography), it has become possible to objectively quantify RNFL thinning, which may appear before manifest field defects on WWP. ${ }^{3,4}$ The objective quantification with photography is possible but tedious, time consuming and requires training.

\section{Currently following Techniques are Available for Structural Assessment of Optic Disk and RNFL}

1. Optic disk photography

2. Retinal nerve fiber layer photography
3. Optical coherence tomography (OCT) (Fig. 1A)

4. Scanning laser polarimetry (GDx VCC) (Fig. 1B)

5. Confocal scanning laser ophthalmoscopy (HRT) (Fig. 1C)

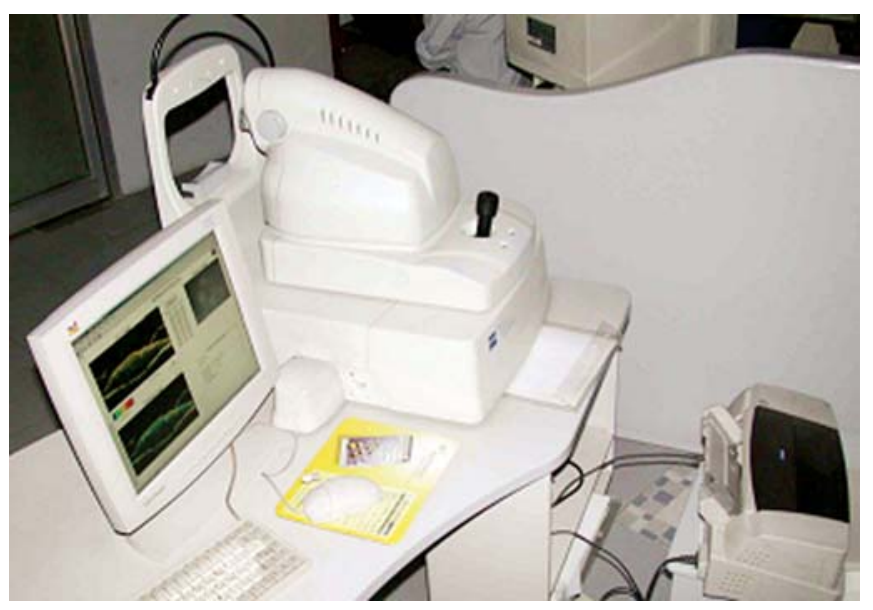

Fig. 1A

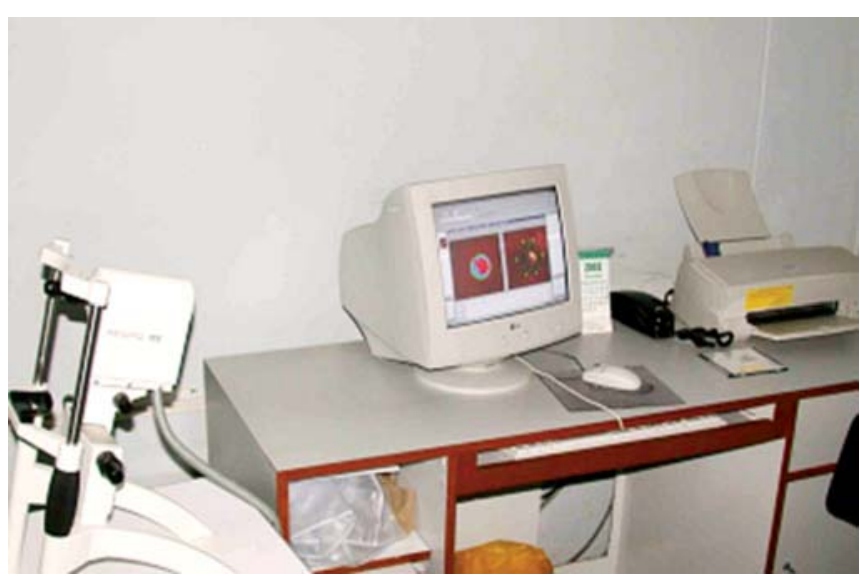

Fig. 1B 


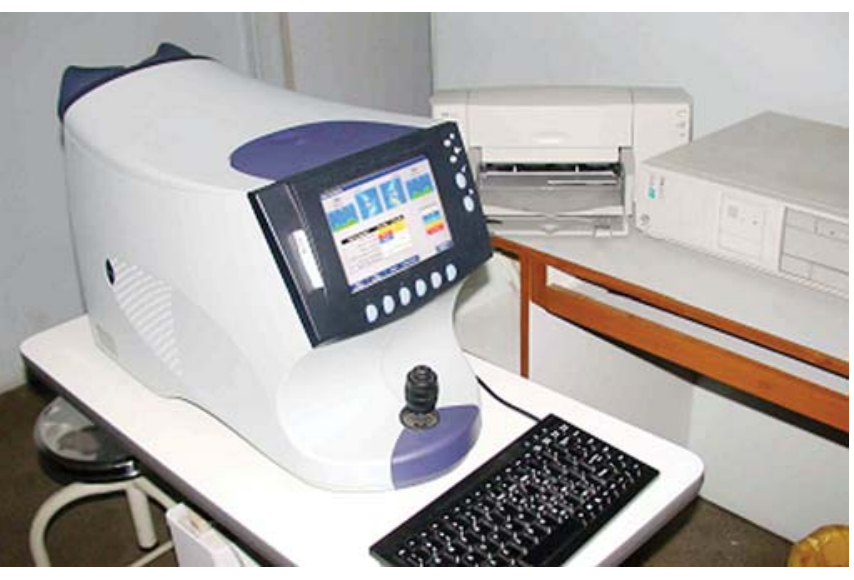

(Fig. 1C)

Figs $1 \mathrm{~A}$ to $\mathrm{C}$ : Different optic nerve head and RNFL imaging modalities

In this article, we will limit our discussion to newer imaging technologies namely HRT, GDx VCC and OCT. In this article, we will discuss only basic of imaging for diagnosis.

\section{Heidelberg Retinal Tomogram (HRT II / HRT III)}

HRT is based on confocal scanning laser ophthalmoscopy principle. This technique allows acquisition of two-dimensional images of the optic nerve head $(\mathrm{ONH})$ and reconstruct into three-dimensional image. The fundus is scanned by a low-power laser beam $(670 \mathrm{~nm})$. The light that reflects back from the scanned points is detected by a photodetector. The images formed from this detection are digitized and stored on computer.

In HRT, sequential confocal scans are acquired in depth at the level of the $\mathrm{ONH}$, and then merged by the in-built software into a 3D image. Measurements are made from this image. The current models are HRTII and HRTIII, which acquire a series of up to 64 optical sections in depth at intervals of $1 / 16 \mathrm{~mm}$.

For analysis, a contour line should be manually placed by the operator to demarcate the limits of the ONH. The in-built software then places an inferior limit which is parallel, and in depth, to the retinal surface. This limit is termed Reference Plane and is placed 50 microns below the contour line. Measurements are made based on this imaginary plane. Figure 2 shows the relationship between the $\mathrm{ONH}$, the contour line and the reference plane.

Figure 3 shows HRT II printout and Figure 4 shows HRT III printout. The printout provides topographic measurements and a classification of the parameters based on a comparison with a normative database. In HRT III, the optic disk parameters are compared with ethnic based normative database (Fig. 4).
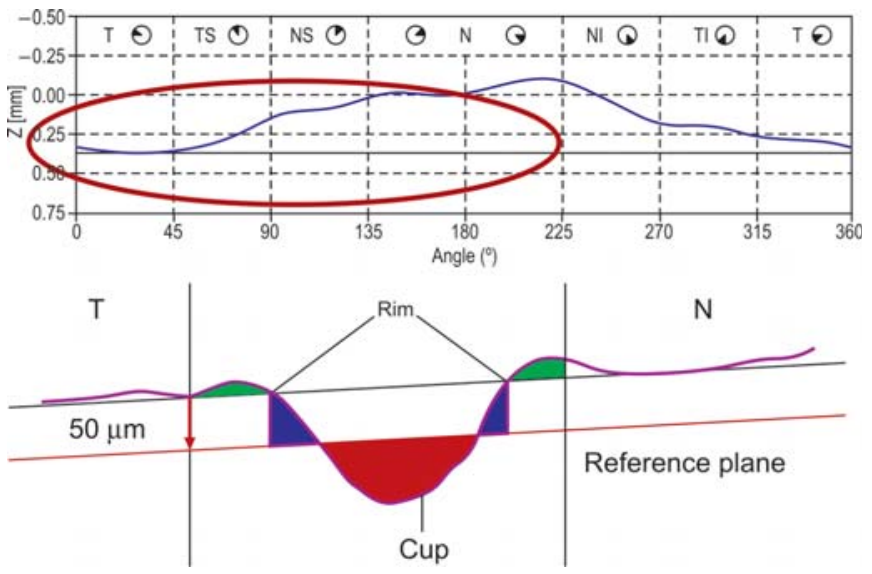

Fig. 2: Relationship between the $\mathrm{ONH}$, the contour line and the reference plane

\section{Parameters Table}

This table shows the parameters measured from an acquired scan. A normal range is also provided. This normal range is calculated from a normative database, which is different from that used for Moorfields Regression Analysis. The HRT III has an ethnic specific database, which includes Indian data also. It also provides statistical value comparing patient result to normative database. Here, $\mathrm{p}<0.05=$ Borderline, $\mathrm{p}<0.001=$ Outside Normal Limits.

1. Patient and exam data: This data includes: name, date of birth, ID number, gender and scan date. Scan data are also provided: focus, depth and operators' initials. We can also include IOP.

2. Quality score: It is a Topography Standard Deviation and indicates quality of an image. (A value $<40$ usually indicates good-quality images).

3. Mean height contour graph: This available in HRT II printout. The green line in Figure 2 (upper image) represents the retinal surface height profile along the contour line. The red line (Fig. 2, lower image) represents the reference plane. The dark black line parallel to the reference plane (Fig. 2, lower image) represents the mean peripapillary retinal surface height.

4. Horizontal height profile: This available in HRT II printout. Horizontal profile represents the height profile along the white horizontal lines across the $\mathrm{ONH}$ in the topography image. The parallel red line on the graph represents the reference plane and the two perpendicular black lines represent the borders of the disc as defined by the contour line previously drawn.

5. Vertical height profile: This available in HRT II printout. Vertical profile has the same characteristics as 'horizontal 
Patient:
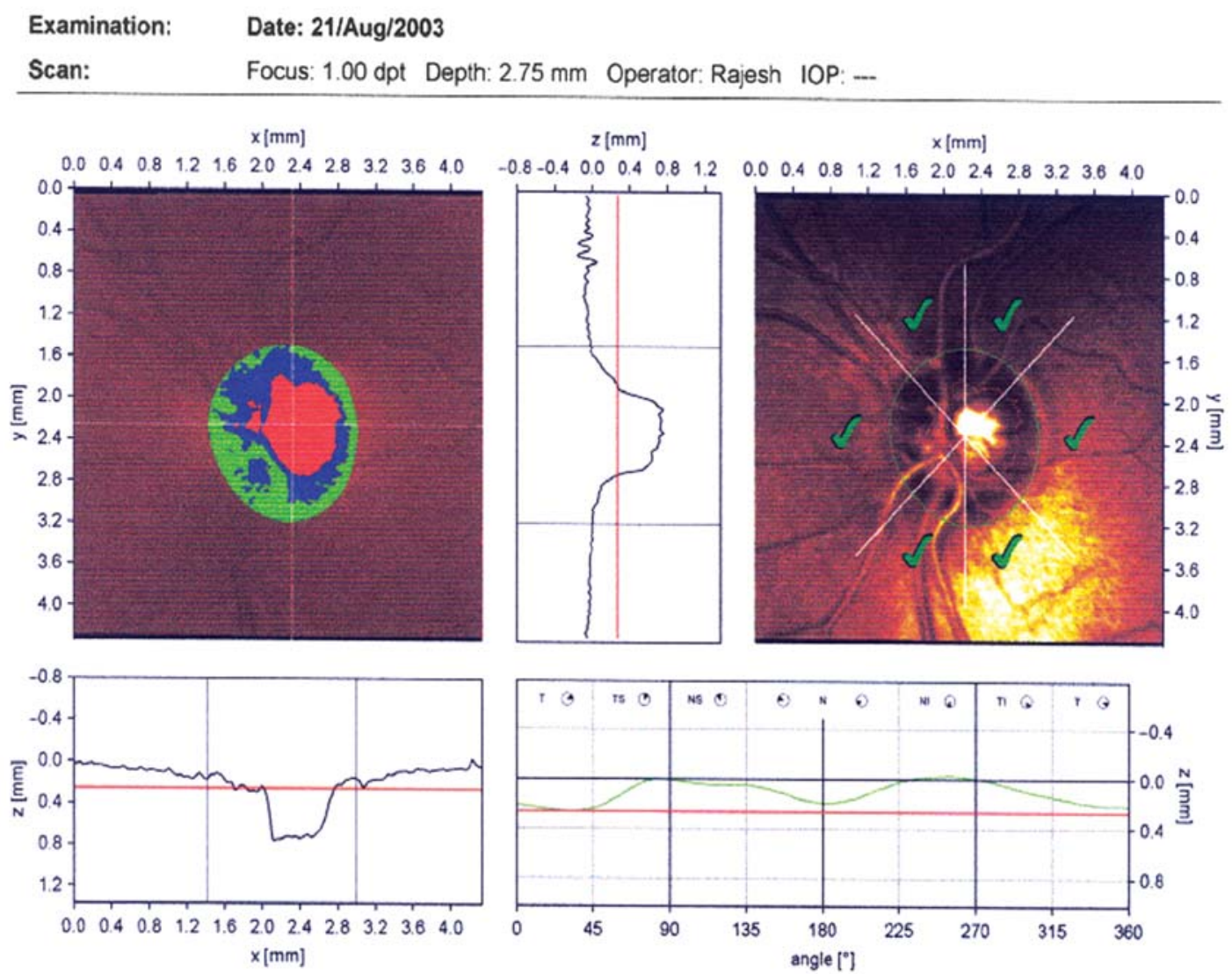

\begin{tabular}{|c|c|c|c|}
\hline Stereometric analysis $\mathrm{ONH}$ & & Normal range & 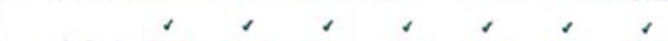 \\
\hline Disk area & $2.133 \mathrm{~mm}^{2}$ & $1.69-2.82$ & \\
\hline Cup area & $0.598 \mathrm{~mm}^{2}$ & $0.26-1.27$ & $\min 20$ \\
\hline Rim area & $1.535 \mathrm{~mm}^{2}$ & $1.20-1.78$ & 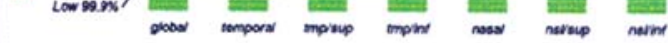 \\
\hline Cup volume & $0.156 \mathrm{cmm}$ & $-0.01-0.49$ & \\
\hline Rim volume & $0.234 \mathrm{cmm}$ & $0.24-0.49$ & \multirow{3}{*}{ 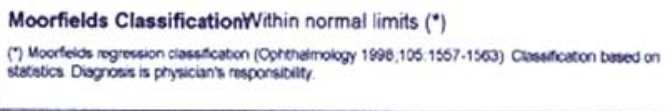 } \\
\hline Cup/disk area ratio & 0.280 & $0.16-0.47$ & \\
\hline Linear cup/disk ratio & 0.529 & $0.36-0.80$ & \\
\hline Mean cup depth & $0.211 \mathrm{~mm}$ & $0.14-0.38$ & \multirow{7}{*}{ Comments: } \\
\hline Maximum cup depth & $0.634 \mathrm{~mm}$ & $0.46-0.90$ & \\
\hline Cup shape measure & -0.226 & $-0.27--0.09$ & \\
\hline Height variation contour & $0.295 \mathrm{~mm}$ & $0.30-0.47$ & \\
\hline Mean RNFL thickness & $0.155 \mathrm{~mm}$ & $0.18 \cdot 0.31$ & \\
\hline RNFL cross-sectional area & $0.801 \mathrm{~mm}^{2}$ & $0.95-1.61$ & \\
\hline Reference height & $0.263 \mathrm{~mm}$ & & \\
\hline Topography std dev. & $12 \mu \mathrm{m}$ & & Date: 04/Aug/2004 Signature: \\
\hline
\end{tabular}

Fig. 3: HRT II printout

profile' but the height profile represented is along the vertical lines across the $\mathrm{ONH}$ in the topography image.

6. Reflection image: It is a 'false' color picture representing changes in reflectivity for each region of the ONH. It is divided into six sectors, which are compared with a normative database, using the Moorfields regression analysis software. This comparison takes into account the relationship between $\mathrm{ONH}$ size and rim area, and 


\section{Heidelberg Retina Tomograph}

OU Report

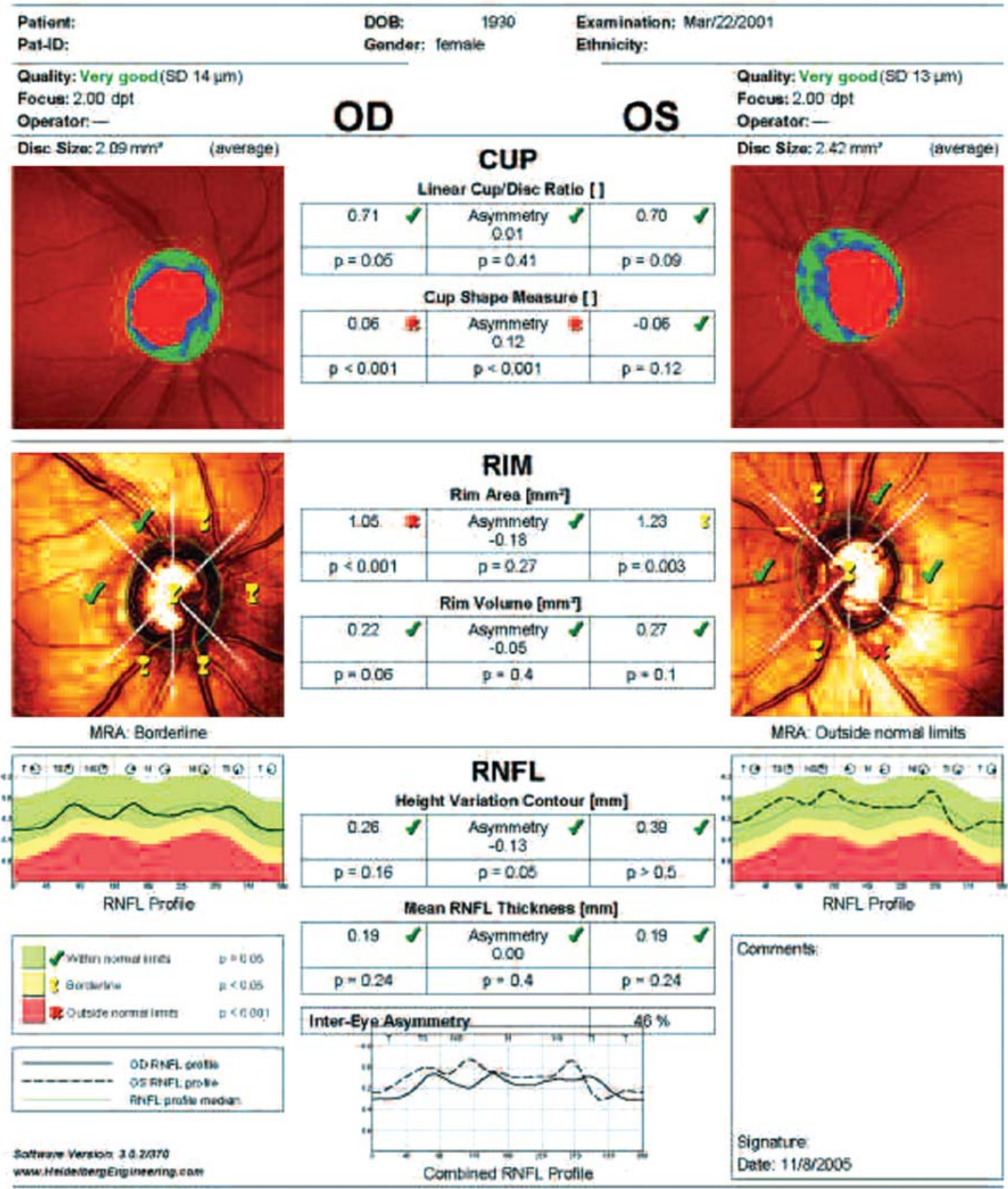

Fig. 4: HRT III printout

classifies each sector as ‘within normal limits’ (green tick), 'borderline' (yellow exclamation mark) or 'outside normal limits' (red cross).
7. Topography image: Topography image is a 'false' color image representing the height of the scanned area (the brighter the color the more depressed the region, and vice 
versa). On the ONH, 'cup area' is seen in red and 'rim area' in blue and green (the difference in color denotes difference in height levels).

8. Parameters: In HRT II printout provides all optic disk and RNFL parameters. In HRT III, along with optic disk size, the software also identifies disk as "small”, "average”, or "large".

9. RNFL profile graph: This is available in HRT III printout. It displays the height values at the optic disk margin going around the optic disk from the temporal side, to superior, nasal, inferior, and back to temporal (TSNIT). The green shaded area is the normal range, yellow is borderline, and the red zone indicates outside normal limits compared to the selected ethnic-specific normative database.

10. Inter-eye asymmetry: It evaluates the symmetry of the RNFL profile between eyes. If the correlation between eyes is good, the value will be near $0 \%$.

Following are the most important diagnostic criteria for HRT.

1. Moorfields regression analysis ${ }^{5}$ (Fig. 5): The column height represents the $\mathrm{ONH}$ (or sector) area. It is divided into the percentage of rim area (green) and percentage of cup area (red). This is compared with age-matched data.

If the percentage of the rim is:

- Larger than or equal to the 95\% limit, the respective sector is classified as 'within normal limits'. This would be marked as a green tick.

- Between the 95\% and the 99.9\% limits, the respective sector is classified as ‘borderline'. This would be marked as a yellow exclamation mark.

- Lower than the 99.9\% limit, the respective sector is classified as 'outside normal limits'. This would be marked as a red cross.

In the HRT printout there is a classification for the whole disc (1st column) and a classification for each single sector (2nd-7th column).
2. FSM discriminant function ${ }^{6}$ : It was developed by Mikelberg and coworkers. The discriminant function is a linear combination of the three parameters cup shape measure, rim volume and contour line height variation (Fig. 6). An eye is classified as being normal if the discriminant function value $\mathrm{F}$ is positive; it is classified as glaucomatous if $\mathrm{F}$ is negative. Mikelberg FS, et al reported sensitivity of $87 \%$ and a specificity of $84 \%{ }^{6}$

3. RB discriminant function: This was developed by Burk R, et al. This discriminant function has high specificity with poor sensitivity for diagnosis of early glaucoma.

4. Ranked sector distribution curves: Here, the optic nerve head is divided into 36 sectors, each $10^{\circ}$ wide, to compute the stereometric parameter values in each segment, and to sort these 36 values in descending order. The result is a graphical representation of the optic nerve head configuration similar to the Bebie curves used in perimetry. From a normal population data, normal RSD curves; the 5th and 95th percentile curves has been calculated. To test a specific eye, its RSD curve is simply plotted together with the normal RSD curves. This is not widely used for diagnosis.

5. Glaucoma probability score ${ }^{8}$ (GPS): GPS utilizes large, ethnic-selectable databases and artificial intelligence (Relevance vector machine), which derives the probability of damage consistent with glaucoma. The GPS provides immediate results without drawing contour lines or relying on reference planes. It has similar sensitivity and specificity to the Moorfields Regression Analysis. This 3D model combines the optic disk topographic information with the peripapillary RNFL information. The model uses five parameters: three characterize the shape of the optic disk (capturing information from the cup size, depth and slope of the rim); two characterize the RNFL (capturing information from the RNFL curvature both horizontally and vertically across the image). A normal result is displayed

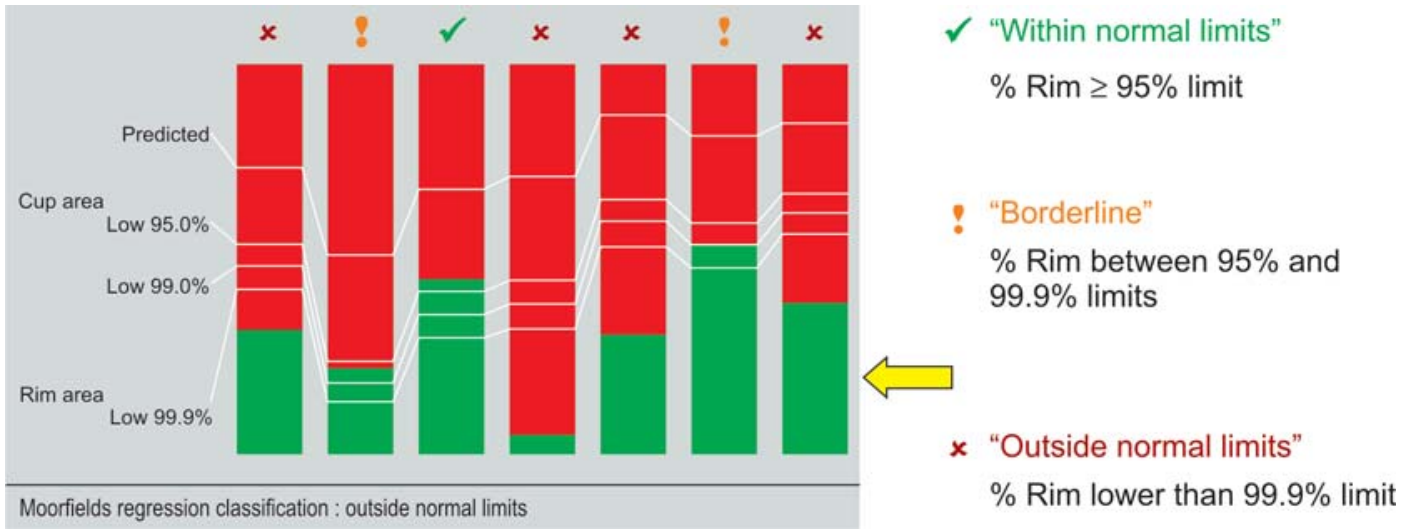

Fig. 5: Shows classification and confidence limit for Moorfields regression analysis 


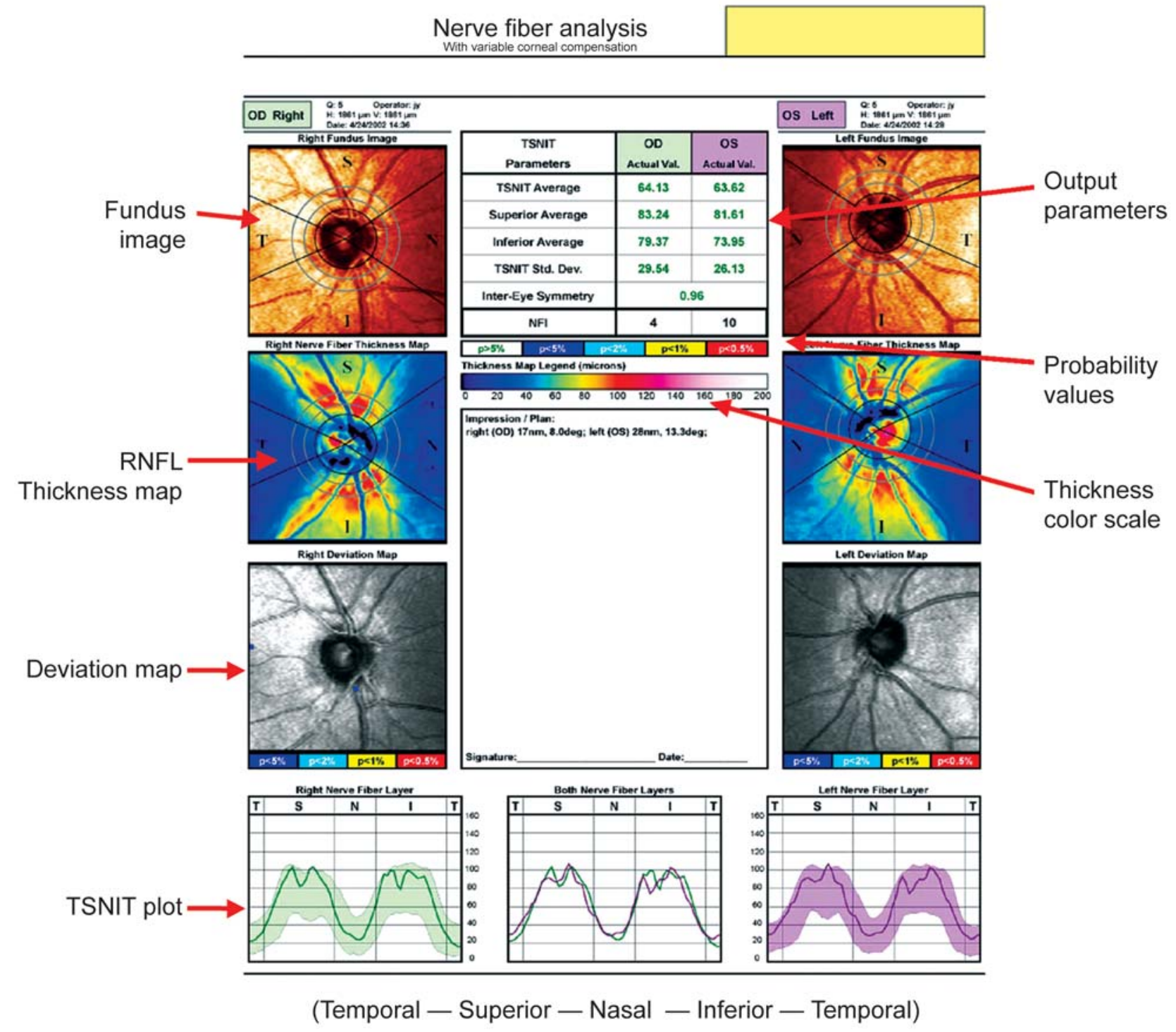

Fig. 6: GDx VCC printout

using a green tick, a borderline result using a yellow exclamation point, and an abnormal result using a red X.

Diagnostic accuracy: HRT measurements have been shown to have high diagnostic accuracy for detecting glaucoma. ${ }^{5,7,8}$ The Moorfields Regression Analysis had a sensitivity and specificity of $84 \%$ and $96 \%$ respectively. ${ }^{5}$ An analysis based on the shape of the optic disk and surrounding RNFL resulted in a sensitivity and specificity of $89 \%$ and $89 \%{ }^{7}$ GPS analysis has a sensitivity and specificity of $91 \%$ and $90 \%{ }^{8}$

In summary, the HRT has in-built software that allows overall diagnostic classification and provides measurement protocols for follow-up analysis. A normative database is incorporated into the software, allowing comparisons with age-matched controls to be made. With the HRT, observer's input is needed for drawing the contour line.

\section{Scanning Laser Polarimetry (GDx VCC)}

The GDx VCC is a confocal scanning laser ophthalmoscope with integrated polarimeter, which uses a near-infrared laser beam $(780 \mathrm{~nm})$ to scan the retina.

The principle of scanning laser polarimetry is to assess RNFL thickness according to light retardation. This involves scanning the retina with a laser beam, which passes through it and reflects from the deeper layers towards the device that releases the laser beam. The retardation of the reflected light is then measured for an estimation of RNFL thickness. 
Figure 6 shows GDx VCC printout. The different features are:

1. Patient and exam data: This contains information about the patient, acquisition site, ID number, name and surname, gender, ancestry and date of birth are mandatory inputs.

2. Image quality: The image quality is shown in the box above the fundus image (scale 1 to 10). The score of 7 or more is desirable.

3. Fundus image: The fundus image is used to check image quality, focus and illumination, and centring of the 'ellipse' (black ring around $\mathrm{ONH}$ ). The ellipse is automatically placed around the optic nerve. In case it is misplaced, its size and position can be easily modified to allow a correct placement. A correctly centered ellipse allows the 'calculation circle' (area between the gray rings) to be placed at the peripapillary area.

4. Thickness map: The thickness map is a color-coded representation of RNFL thickness. Thick RNFLs are colored in yellow, orange and red (the warmer the color, the thicker the RNFL), while thin RNFL values are colored dark and light blue (the deeper the blue, the thinner the RNFL). Generalized RNFL loss results in a more uniform blue appearance, and a focal (wedge) defect appears as a dark blue band originating at the $\mathrm{ONH}$, extending through the bundle.
5. Deviation map: The deviation map reveals the location and severity of RNFL defects over the entire thickness map. It analyses a $20 \times 20$ degree region centered on the optic disk using a gray scale fundus image of the eye as a background. The map is averaged into a grid of $32 \times 32$ squares, where each square (super pixel) is compared with the age-matched normative database. Super pixels that fall below the normal range are flagged by colored squares, representing varying probabilities of normality.

6. TSNIT curve: TSNIT stands for Temporal-Superior-NasalInferior-Temporal. The graph displays RNFL thickness values calculated within the calculation circle, starting temporally and moving superiorly, nasally, inferiorly, and ending temporally for both eyes (darker line). The shaded area represents the normal range for that age.

7. Parameters table (Fig. 7)

a. TSNIT average: It is the average of the RNFL thickness within the calculation circle.

b. Superior averages: This is the thickness measured within the superior $120^{\circ}$

c. Inferior averages: This is the thickness measured within the inferior $120^{\circ}$.

d. The TSNIT Std. Dev: It represents the standard deviation of the overall measurement: the bigger the number, the healthier the eye.

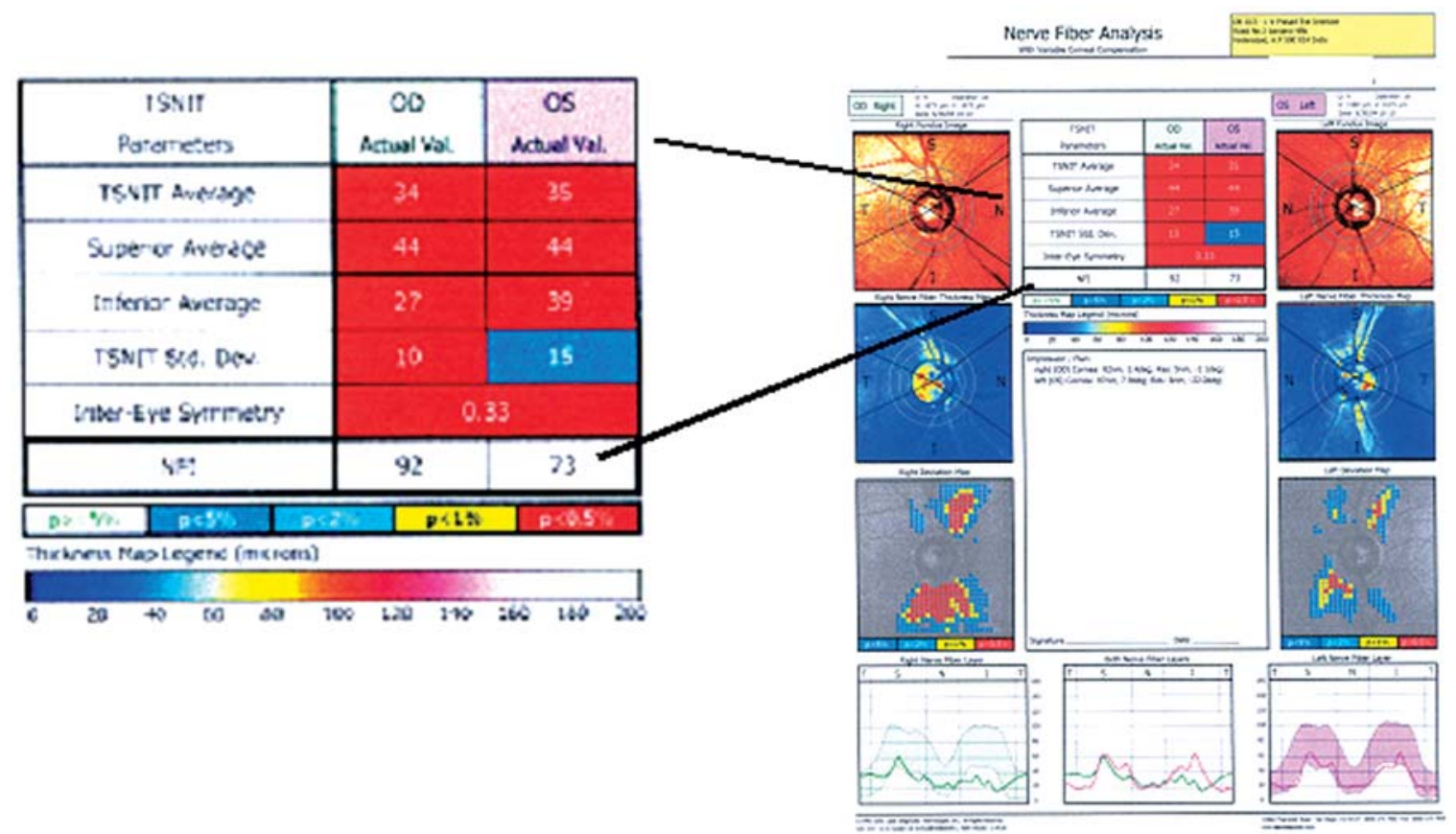

- Parameters-Summary measures based on the calculation circle

- Values outside normal are color-coded based on probability of abnormality

Fig. 7: Global parameters 
e. Inter-eye symmetry: It is a correlation coefficient of the total measurement from both eyes. A value close to 1 means high symmetry between eyes.

f. NFI (Nerve Fiber Indicator): It is an indicator of the likelihood of an eye to have glaucoma. It is calculated with an artificial intelligence software using data obtained from within and outside the calculation circle.

\section{Nerve Fiber Index}

The nerve fiber index (or NFI) is based on an advanced form of neural network analysis, which is trained to differentiate optimally normal from glaucomatous eyes. The entire RNFL profile is analyzed, and the diagnostic classification of the output is determined using the normative database (data from normal eyes). The 95th percentile is used as the cut-off for classifying the RNFL as 'normal' (between 0 and 30) or 'borderline' (between 31 and 50). The 99th percentile is used as the cut-off for classifying the RNFL as 'glaucomatous' (between 51 and 100). ${ }^{9}$

\section{Diagnostic Accuracy}

The reported sensitivity and specificity of GDx in the early diagnosis of glaucoma ranges from 72 to $78 \%$ and 56 to $92 \%$ respectively. ${ }^{10-14}$ In literature, most of researchers have not find any single parameter with high diagnostic accuracy. We feel that the use of combination of more than one parameter can be used to improve the diagnostic accuracy of GDx VCC. For example, NFI >50 has maximum specificity, positive likelihood ratio. If positive, it can be used to virtually 'rule in' the disease. $\mathrm{NFI}<20$ has maximum sensitivity and negative likelihood ratio. If negative it can be used to 'rule out' the disease.

\section{Future Potential}

The ECC (Enhanced Corneal Compensation) is a software solution to reduce the atypical pattern that can be found in some scans. The ECC will allow the reduction of noise in scans.

In summary, the GDx VCC is a new technology, which has in-built software which allows overall diagnostic classification. A normative database is incorporated into the software, allowing comparisons with age-matched controls to be made.

\section{OPTICAL COHERENCE TOMOGRAPHY (OCT)}

OCT can be used to scan the ONH, peripapillary retina and macular region. An 820-nanometer near-infrared light beam is used, and the reported depth resolution is less than or equal to 10 microns. $^{16-20}$

It is a noncontact, noninvasive imaging technique that provides high-resolution cross-sectional images of the retina. The principle is similar to ultrasound but here light is used to scan the retina. The intensity of light signal reflecting from retinal structures is used to create a tomographic image. The combined use of low-coherence light and an interferometer provides high depth resolution, which yields near-histological differentiation of the structures studied. This is based on Michelson interferometer principle.

OCT has optic nerve head analysis, RNFL analysis and macular parameter analysis.

1. Printout - ONH useful parameters (Fig. 8)

The useful parameters for an OCT printout of the ONH are:

- The raw and composite ONH scans. The composite scan is obtained from 6 to 24 scans.

- $\quad$ The individual and global stereometric parameters.

2. Printout - RNFL useful parameters (Fig. 9)

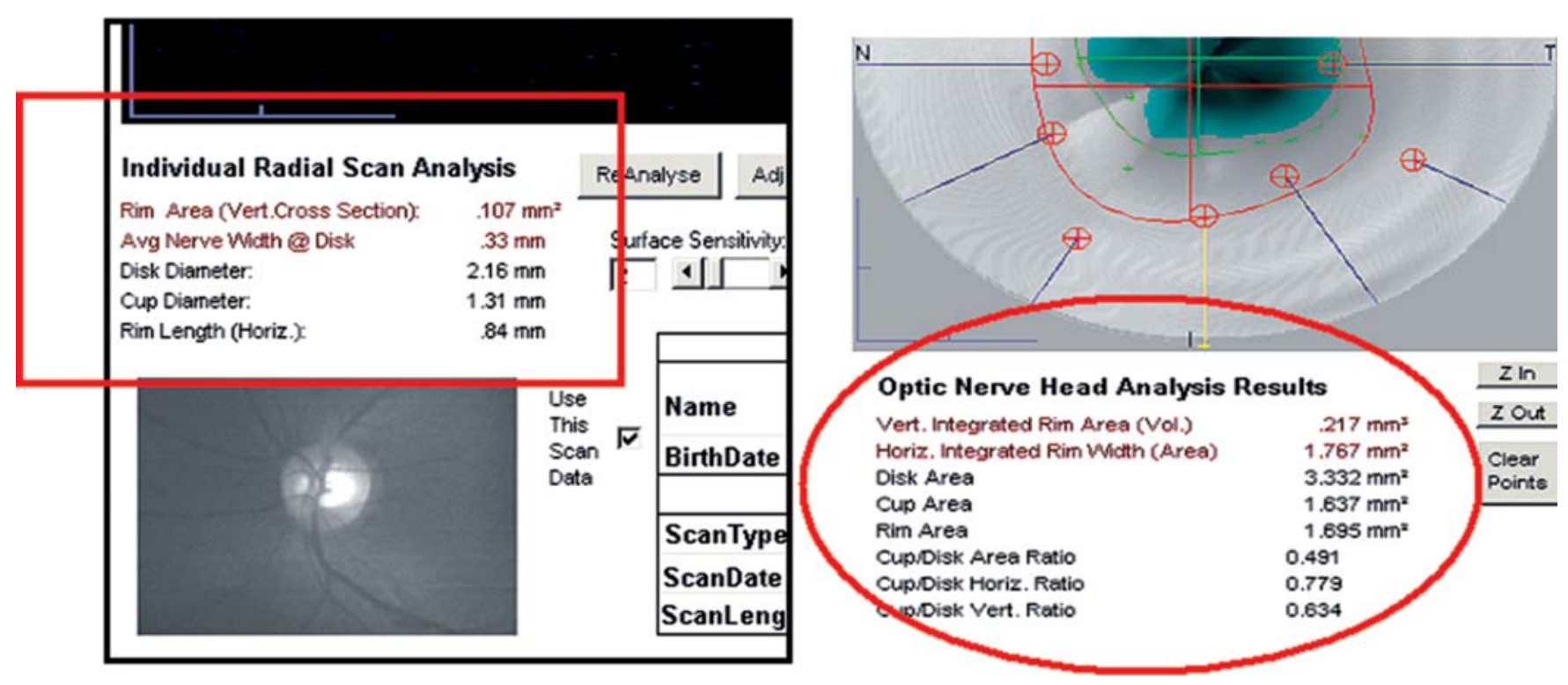

Fig. 8: Optic nerve head analysis with stratus OCT 3 


\section{STRATUS OCT \\ RNFL Thickness Average Analysis Report - Ver. 3.0}
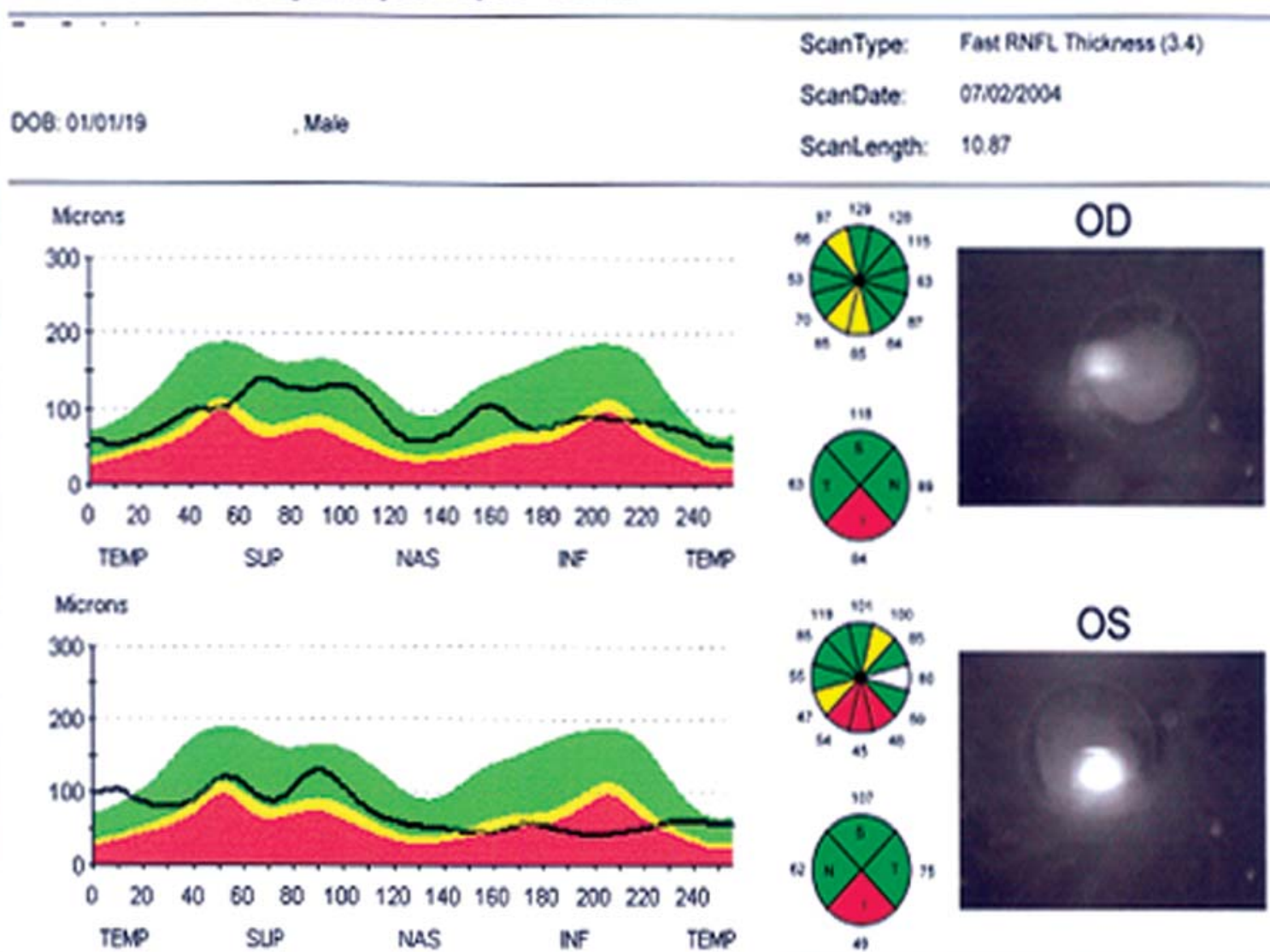

Morons
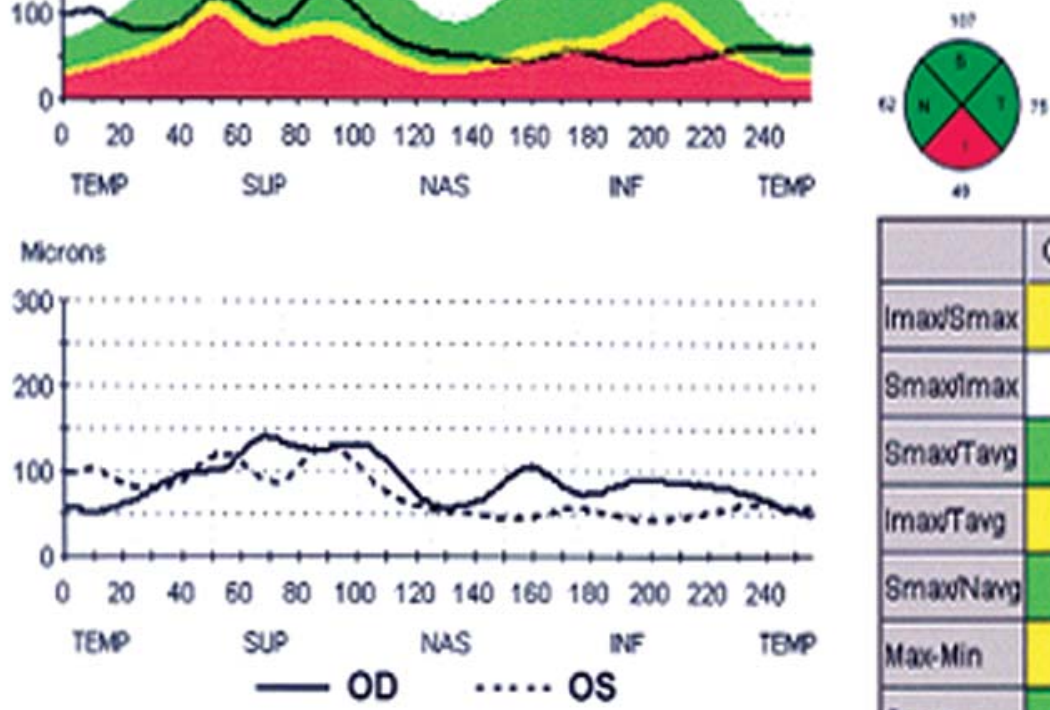

\begin{tabular}{|c|c|c|c|}
\hline & $O D(N=3)$ & $08(N=3)$ & OD.08 \\
\hline imatsmax & 0.73 & 0.44 & 0.29 \\
\hline Smaximax & 1.37 & 2.28 & $\cdot 0.91$ \\
\hline Smavtang & 2.23 & 1.75 & 0.48 \\
\hline imartang & 1.62 & 0.77 & 0.86 \\
\hline Smaving & 1.59 & 2.11 & $\cdot 0.52$ \\
\hline MaxeMin & 91.00 & 90.00 & 1.00 \\
\hline $8 m x x$ & 140.00 & 131.00 & 9.00 \\
\hline imax & 02.00 & 58.00 & 44.00 \\
\hline Savg & 18.00 & 107.00 & 11.00 \\
\hline $\operatorname{lom} 8$ & 84.00 & 49.00 & 3500 \\
\hline Ang. Thick & 88.46 & 73.14 & 15.32 \\
\hline
\end{tabular}

Fig. 9: OCT printout (RNFL analysis) 

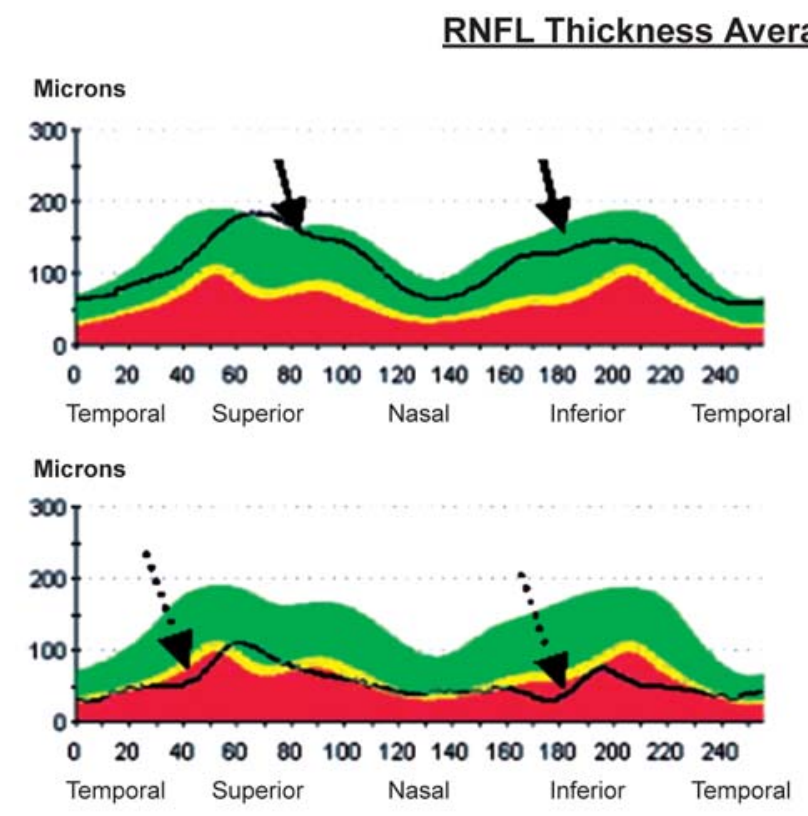

OS
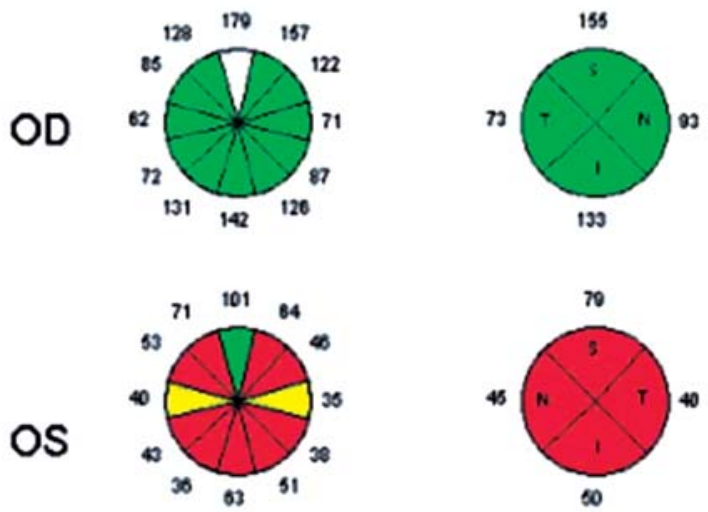

\begin{tabular}{|c|c|}
\hline \multirow{2}{*}{\multicolumn{2}{|c|}{$\begin{array}{l}\text { Patient/Scan Information } \\
\text { PD. } \infty \text { Normal } \\
\text { OS Early Gloucorna } \\
\text { DOE: 06/23/1979, ID. NA, Msle }\end{array}$}} \\
\hline & \\
\hline ScanType & Fast RNFL Thickness (3.4) \\
\hline ScanDate & $01 / 29 / 2002$ \\
\hline ScanLength & 10.87 \\
\hline
\end{tabular}

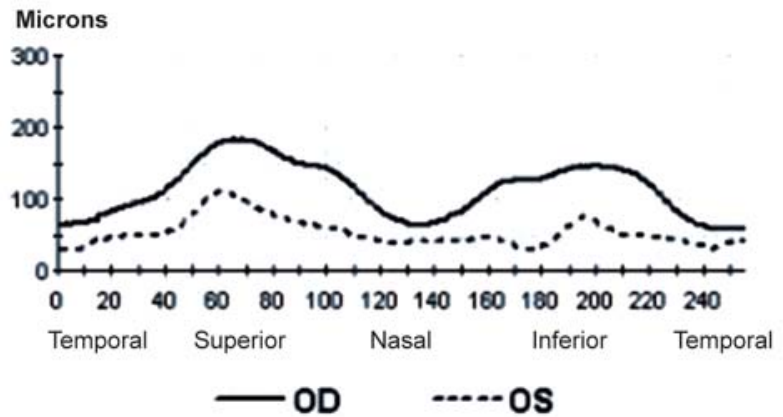

\begin{tabular}{|c|c|c|c|}
\hline & $\infty O(N=3)$ & $\cos (\mathrm{N}=3)$ & 00.05 \\
\hline Inacismax & 0.80 & 0.67 & 0.13 \\
\hline Simaxdmax & 125 & 1.50 & -0.24 \\
\hline Smaxrfavg & 2.49 & 2.78 & -0.29 \\
\hline insoffavg & 1.99 & 1.86 & 0.13 \\
\hline SmbociNavg & 1.96 & 2.43 & -0.47 \\
\hline Max-Mn & 126.00 & 82.00 & 44.00 \\
\hline Sinax & 183.00 & 110.00 & 73.00 \\
\hline $\ln x x$ & 146.00 & 74.00 & 72.00 \\
\hline Savg & 155.00 & 79.00 & 76.00 \\
\hline levg & 133.00 & 50.00 & 83.00 \\
\hline Avg. Thickness & 113.60 & 53.57 & 60.03 \\
\hline
\end{tabular}

Fig. 10: RNFL thickness average analysis

The useful parameters for an OCT printout of the RNFL are:

- Total, quadrant and clock-hour sectors of RNFL thickness. Clock-hour sectors consist of 12 equal 30degree sectors.

- An in-built normative database allows the comparison between acquired measurements and data and normal, age-matched data. Comparison is made if one of the sector type is selected.

- The differences between the two eyes (inter-eye differences), as both eyes are measured.

As RNFL analysis is widely reported and used, we will limit our discussion to RNFL analysis.

\section{Printout - RNFL}

1. Patient and exam data: This includes name, date of birth, gender and ID number. It also includes scan protocol used, date of the scan and analysis protocol selected.
2. Fundus photography: It is obtained immediately after scanning, and is used to evaluate the correct position of the circular scan around the $\mathrm{ONH}$.

3. Signal strength: The signal strength is shown in the box on the right (scale 1 to 10). The signal strength of 7 or more is desirable.

4. RNFL thickness analysis (both eye): RNFL profile is a graphic representation of the RNFL thickness around the $\mathrm{ONH}$. On the X-axis, the sector of the RNFL is represented according to the temporal-superior-nasal-inferior-temporal sequence: the circular scan is linearly represented, starting from the temporal region, continuing towards the superior, nasal, inferior regions, and terminating in the temporal region. On the Y-axis, thickness is expressed in microns. (Fig. 10). The patient's RNFL graph is compared with age matched normative database. 
RNFL thickness measurements made around the $\mathrm{ONH}$ for each eye are presented as two circles: the top one is divided into 12 o' clock-hour sectors and the bottom one into four equal $90^{\circ}$ sectors. Each sector is represented with a number and color code. The number represents the thickness expressed in microns, and the color code indicates the possibility of that value being found in the normal population:

- Green and white: values found in up to 95\% of the normal population.

- Yellow: values found in less than 5\% of the normal population.

- Red: values found in less than $1 \%$ of the normal population.

6. Parameters table: The parameters table presents data from both eyes plus inter-eye differences. Color indicates the probability of finding similar values in a normal population. The color coding and its statistical significance are same as discussed above in RNFL profile.

\section{DIAGNOSTIC ACCURACY}

Several studies have evaluated the diagnostic ability of OCT parameters for glaucoma. In most of the published literature, RNFL thickness in the inferior region often had the best ability to discriminate healthy eyes from those with glaucoma, with sensitivities ranging between $67 \%$ and $84 \%$ for specificities of more than 90\%. In our study, we have reported 6-clock, RNFL thickness parameter with very high positive likelihood ratio, which can virtually rule in the disease if positive.

\section{FUTURE POTENTIALS}

There are various other newer models of OCT are under research (En-Face OCT, High-speed ultra-high resolution OCT, Spectraldomain OCT, Polarization-sensitive OCT, Functional ultra-high resolution OCT). ${ }^{21-26}$

In summary, OCT is an evolving technology, and more powerful tools based on this technology are being developed. A normative database is incorporated into the software, allowing comparisons with age-matched controls to be made.

\section{REFERENCES}

1. Quigley HA, Addicks EM. Quantitative studies of retinal nerve fiber layer defects. Arch Ophthalmol 1982;100:807-14.

2. Quigley HA, Dunkelberger GR, Green WR. Retinal ganglion cell atrophy correlated with automated perimetry in human eyes with glaucoma. Am J Ophthalmol 1989;15:107:453-64.

3. OCT manual, Carl Zeiss Meditac, Dublin, CA, 2004.

4. Quigley HA, Dunkelberger GR, Baginski TA, et al. Chronic human glaucoma causing selectively greater loss of larger optic nerve fibers. Ophthalmology 1988;95:357-63.
5. Wollstein G, Garway-Heath DF, Hitchings RA. Identification of early glaucoma cases with the scanning laser ophthalmoscope. Ophthalmology 1998;105:1557-63.

6. Iester M, Mikelberg FS, Drance SM. The effect of optic disc size on diagnostic precision with the Heidelberg Retina Tomograph. Ophthalmology 1997;104:545-48.

7. Swindale NV, Stjepanovic G, Chin A, Mikelberg FS: Automated analysis of normal and glaucomatous optic nerve head topography images. Invest Ophthalmol Vis Sci 2000;41:173042.

8. Bowd C, Chan K, Zangwill LM, Goldbaum MH, Lee T, Sejnowski TJ, Weinreb RN. Comparing neural networks and linear discriminant functions for glaucoma detection using confocal scanning laser ophthalmoscopy of the optic disc. Invest Ophthalmol Vis Sci 2002;43:3444-54.

9. Reus NJ, Lemij HG. Ophthalmology 2004;111:1860-65.

10. Bowd C, Zangwill LM, Berry CC, et al. Detecting early glaucoma by assessment of retinal nerve fiber layer thickness and visual function. Invest Ophthalmol Vis Sci 2001;42:1993-2003.

11. Da Pozzo S, Fuser M, Vattovani O, et al. GDx-VCC performance in discriminating normal from glaucomatous eyes with early visual field loss. Graefes Arch Clin Exp Ophthalmol 2006;244: 689-95.

12. Brusini P, Salvetat ML, Parisi L, et al. Discrimination between normal and early glaucomatous eyes with scanning laser polarimeter with fixed and variable corneal compensator settings. Eur J Ophthalmol 2005;15:468-76.

13. Tjon-Fo-Sang MJ, Lemij HG. The sensitivity and specificity of nerve fiber layer measurements in glaucoma as determined with scanning laser polarimetry. Am J Ophthalmol 1997;123:62-69.

14. Parikh RS, Parikh SR, Chandra Sekhar G, Prabakaran, SJ Ganesh babu, Thomas R. Diagnostic capability of scanning laser polarimetry (GDx VCC) in early glaucoma. Ophthalmology 2007 Nov 29; [Epub ahead of print].

15. Schuman JS, Hee MR, Puliafito CA, et al. Quantification of nerve fiber layer thickness in normal and glaucomatous eyes using optical coherence tomography. Arch Ophthalmol 1995;113:586-96.

16. Nouri-Mahdavi K, Hoffman D, Tannenbaum DP, et al. Identifying early glaucoma with optical coherence tomography. Am J Ophthalmol 2004;137:228-35.

17. Bowd C, Zangwill LM, Berry CC, et al. Detecting early glaucoma by assessment of retinal nerve fiber layer thickness and visual function. Invest Ophthalmol Vis Sci 2001;42:1993-2003.

18. Budenz DL, Michael A, Chang RT, et al. Sensitivity and specificity of the Stratus OCT for perimetric glaucoma. Ophthalmology 2005;112:3-9.

19. Kanamori A, Nakamura M, Escano MF, et al. Evaluation of the glaucomatous damage on retinal nerve fiber layer thickness measured by optical coherence tomography. Am J Ophthalmol 2003;135:513-20.

20. Parikh RS, Parikh SR, Chandra Sekhar G, Prabakaran, SJ Ganesh babu, Thomas R. Diagnostic Capability of Optical Coherence Tomography (Stratus OCT 3) in Early Glaucoma. Ophthalmology 2007;114(12):2238-43.

21. Drexler W. Ultrahigh-resolution optical coherence tomography. J Biomed Opt. Jan-Feb 2004;9(1):47-74. Review. 
22. Podoleanu AG, Dobre GM, Cucu RG, Rosen R, Garcia P, Nieto J, Will D, Gentile R, Muldoon T, Walsh J, Yannuzzi LA, Fisher Y, Orlock D, Weitz R, Rogers JA, Dunne S, Boxer A. Combined multiplanar optical coherence tomography and confocal scanning ophthalmoscopy. J Biomed Opt 2004;9(1):86-93.

23. Nassif N, Cense B, Park BH, Yun SH, Chen TC, Bouma BE, Tearney GJ, de Boer JF. In vivo human retinal imaging by ultrahigh-speed spectral domain optical coherence tomography. Opt Lett 2004; 29(5):480-82.

24. de Boer JF, Cense B, Park BH, Pierce MC, Tearney GJ, Bouma BE. Improved signal-to-noise ratio in spectral-domain compared with time-domain optical coherence tomography. Opt Lett. 2003;28(21):2067-69.

25. Cense B, Chen TC, Park BH, Pierce MC, de Boer JF. Thickness and birefringence of healthy retinal nerve fiber layer tissue measured with polarization-sensitive optical coherence tomography. Invest Ophthalmol Vis Sci. 2004;45(8):2606-12.

26. Bizheva K, Pflug R, Hermann B, Povazay B, Sattmann H, Qiu P, Anger E, Reitsamer H, Popov S, Taylor JR, Unterhuber A,
Ahnelt P, Drexler W. Optophysiology: depth-resolved probing of retinal physiology with functional ultrahigh-resolution optical coherence tomography. Proc Natl Acad Sci USA 28; 2006;103(13):5066-71.

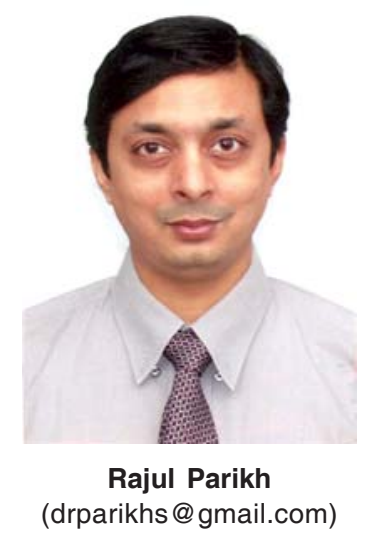

"Each man has only one genuine vocation-to find the way to himself... his task is to discover his own destiny-not an arbitrary one-and live it out wholly and resolutely within himself. Everything else is only a would-be existence, an attempt at evasion, a flight back to the ideals of the masses, conformity and fear of one's own inwardness."

— Demian by Hermann Hesse 